\title{
Distribution of mast cell subtypes in interstitial cystitis: implications for novel diagnostic and therapeutic strategies?
}

\author{
Shabana T Malik, ${ }^{1}$ Brian R Birch, ${ }^{2}$ David Voegeli, ${ }^{1}$ Mandy Fader, ${ }^{1}$ Vipul Foria, ${ }^{3}$ \\ Alan J Cooper, ${ }^{4}$ Andrew F Walls, ${ }^{2}$ Bashir A Lwaleed ${ }^{1}$
}

${ }^{1}$ Faculty of Health Sciences, University of Southampton, Southampton, UK

${ }^{2}$ Faculty of Medicine, University of Southampton, Southampton, UK

${ }^{3}$ Department of Histopathology, University Hospital

Southampton NHS Foundation Trust, Southampton, UK

${ }^{4}$ Faculty of Pharmacy and Biomedical Sciences, University of Portsmouth, Portsmouth, UK

\section{Correspondence to} Associate Professor Bashir A Lwaleed, Faculty of Health Sciences, University of Southampton, Southampton S0166YD,UK; bashir@soton. ac.uk

Received 27 October 2017 Revised 3 April 2018 Accepted 10 April 2018
Check for updates

To cite: Malik ST, Birch BR, Voegeli D, et al. J Clin Pathol Epub ahead of print: [please include Day Month Year]. doi:10.1136/ jclinpath-2017-204881

\begin{abstract}
Aims To identify the presence and geographical distribution of mast cell (MC) subtypes: $\mathrm{MC}_{\mathrm{T}}$ (tryptase positive-chymase negative) and $\mathrm{MC}_{\mathrm{TC}}$ (tryptase positivechymase positive) in bladder tissue.

Methods Bladder tissue was obtained from patients with painful bladder syndrome/interstitial cystitis $(n=14)$ and normal histology from University Hospital Southampton tissue bank. Sequential tissue slices were immunohistochemically stained for MC subtypes using anti-MC tryptase (for $\mathrm{MC}_{\mathrm{T}}$ and $\mathrm{MC}_{\mathrm{TC}}$ ) and anti-MC chymase (for $M C_{T C}$ ). Stained sections were photographed, and positively stained MCs were quantified using ImageJ. Data were analysed using descriptive statistics and individual paired t-tests.
\end{abstract}

Results There was a significant difference in the density of MCs between each layer of the disease bladder, with the greatest accumulation within the detrusor $(p<0.001)$. There was a significant increase in $\mathrm{MC}_{\mathrm{TC}}$ subtype in the lamina $(p=0.009)$ in painful bladder syndrome/interstitial cystitis.

Conclusions Our results suggest that mastocytosis is present within all layers of disease bladder, especially the muscle layer. The varying increase in MC subtypes in the lamina and mucosa may explain the variability in painful bladder syndrome/interstitial cystitis symptoms. A high influx of $M C_{T C}$ in the mucosa of individuals who also had ulceration noted within their diagnostic notes may be of the Hunner's ulcer subclassification. These findings suggest a relationship between the pathogenesis of $\mathrm{MC}$ subtypes and the clinical presentation of painful bladder syndrome/interstitial cystitis. A cohort study would further elucidate the diagnostic and/or therapeutic potential of MCs in patients with painful bladder syndrome/ interstitial cystitis.

\section{INTRODUCTION}

Painful bladder syndrome/interstitial cystitis (PBS/ IC) is a chronic inflammation of the urinary bladder, which is difficult to diagnose and treat and has unsatisfactory therapeutic options. The pathogenesis of PBS/IC is largely unknown, and instead it is thought that PBS/IC is caused by a variety of means. Thus, subgroups of PBS/IC patients exist based on their underlying pathology. Although the ulcer and non-ulcer subclassification is currently used, it could be argued that this broad grouping requires further subdividing into groups based on the influx of mast cells (MCs), fibrosis and other pathologies seen in patients with PBS/IC.
PBS/IC sufferers have a defective glycosaminoglycan (GAG) layer, which normally acts as a protective lining over the bladder urothelium. Research has shown that dysfunctional or damaged urothelial cells generate cytokines such as stem cell factor $(\mathrm{SCF})^{2}$ that can promote activation and proliferation of MCs, as bladder MCs are maximally activated by $\mathrm{SCF}^{3}$ Following the leakage of urinary components (especially potassium due to its ability to depolarise nerves ${ }^{4}$ ) through the damaged urothelium, an inflammatory response involving MCs is triggered. ${ }^{5}$ A histological feature of PBS/IC is a redistribution of MCs into the detrusor muscle layer. These redistributed MCs act on local smooth muscle and vascular epithelium and also have a significant effect on inflammation. ${ }^{6}$ Christmas et $a l^{7}$ reported a significant increase in the density of nerve fibres in patients with PBS/IC. Most of these newly proliferated nerve fibres were found to contain substance $\mathrm{P}$ (SP) ${ }^{8}$ a potent stimulator of MC degranulation, and the histamine subsequently released feeds back to induce the release of further SP. ${ }^{9} \mathrm{MC}$ degranulation is triggered by molecules such as SP, SCF and nerve growth factor, factors that have all been implicated in the pathology of PBS/IC. ${ }^{10}$ Once activated, MCs release molecules (such as histamine, interleukin-6 and tumour necrosis factor- $\alpha$ ) that play a key role in MC activation, MC recruitment and inflammation.

MCs express a high level of two serine proteases: chymase and tryptase. To distinguish the location of human MCs, antibodies that target $\mathrm{MC}$ proteases can be used. Tryptase is considered to be an important marker of MC activity and so the antibody AA1 (anti-MC tryptase) can be used to identify tryptase containing MCs (ie, $\mathrm{MC}_{\mathrm{T}}$ and $\left.\mathrm{MC}_{\mathrm{TC}}\right)$. Chymase can be found in $\mathrm{MC}_{\mathrm{TC}}$, and so this MC subtype can be identified using the antibody CC1 (anti-MC chymase). Sparse research exists using antitryptase and antichymase antibodies in the identification of MCs in PBS/IC, ${ }^{11} 12$ and additionally, there is currently no research comparing the densities of MC subtypes in PBS/ IC using a colocalisation technique.

PBS/IC may be associated with the proliferation and activation of MCs in the bladder wall. Thus, the objective of this study was to determine the subpopulations and location of MCs in bladder tissue using antibody-based techniques. 


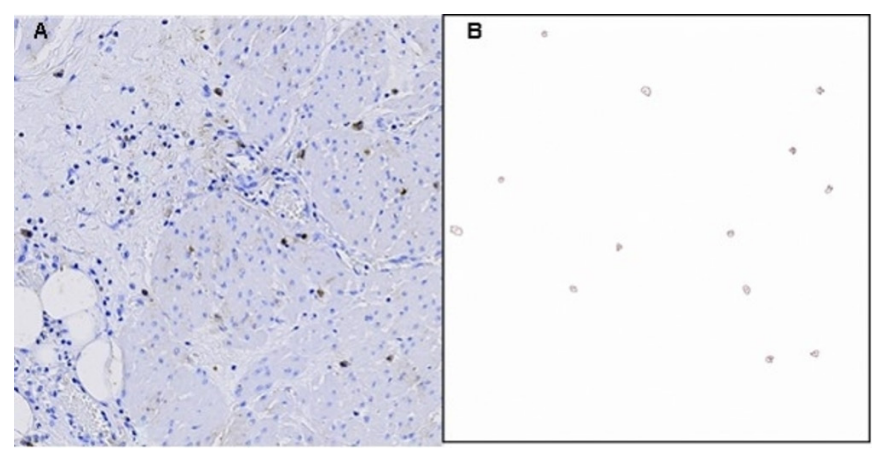

Figure 1 Representative images of AA1 positively stained PBS/ IC bladder tissue. (A) The detrusor muscle layer positively stained for mast cell tryptase using the AA1 antibody (brown). (B) The 'skeleton' left after the image has been processed by ImageJ. The macro has successfully detected all well-defined mast cells. PBS/IC, painful bladder syndrome/interstitial cystitis.

\section{MATERIALS AND METHODS}

Bladder sections were obtained from the tissue bank at the Department of Histopathology, University Hospital Southampton NHS Foundation Trust. The 2008 criteria of the European Society for the Study of Interstitial Cystitis were used. The tissue was originally collected during cystoscopy, with patient consent. Inclusion into this study was based on the amount of tissue present within the block (full-thickness samples were selected, whereas biopsy samples were excluded due to a lack of adequate tissue). The medical history of the subject was also considered; that is, subjects with a histological positive diagnosis of PBS/IC were selected (all other patient information was blinded to the researcher). Patient diagnostic information showed that a positive diagnosis of PBS/ IC was made predominantly based on the presence of inflammation, a prominent infiltration of MCs within the detrusor layer of the bladder wall and denudation of the urothelium. The study included control tissue from subjects that appeared histologically normal and did not show cellular abnormalities associated with inflammation. These controls were individuals who had lower urinary tract symptoms, but had no inflammatory cell infiltrate or other markers of inflammation, on histological examination. Bladder sections were obtained from the tissue bank. Tissue had been fixed in $10 \%$ buffered formalin before a conventional wax embedding process.

Tryptase is present in abundance in all MCs (immune system and non-immune system associated MCs) and is often used as a marker for MC activation. The AA1 antibody is highly sensitive and specific for tryptase, with minimal background staining of other tissue structures and thus is routinely used for the identification of MCs. Chymase is often studied when looking at mastocytosis-associated diseases. This proteinase is less prominent than tryptase, as it is only found in non-immune system associated MCs and is thought to play a key role in fibrosis and smooth muscle degradation. The CC1 antibody is able to detect chymase successfully, with minimal background staining of other tissue structures.

A conventional immunohistochemistry technique was employed. Blocks were cut at $4 \mu \mathrm{m}$, and sequential sections were applied to Apex-coated slides. The slides were dewaxed and rehydrated to $70 \%$ alcohol. A combination of hydrogen peroxidase and methanol was applied to all slides for $10 \mathrm{~min}$ to inhibit exogenous peroxidase and minimise background staining. Slides were then washed with Tris-buffered saline (TBS) before the antigen retrieval step. As MCs are the cell type of interest, a pronase solution was applied for $10 \mathrm{~min}$ in order to unmask antibody binding sites for chymase and tryptase. To further increase the specificity of antigen binding and reduce background staining, endogenous biotin was also blocked by incubating with avidin for $20 \mathrm{~min}$, washing in TBS and then incubating for $20 \mathrm{~min}$ with biotin. The slides were then rinsed before the application of culture medium and left to incubate at room temperature for $20 \mathrm{~min}$. The slides were drained and either the monoclonal antibody AA1 or CC1 was applied at a dilution of either 1:50000 or 1:100, respectively (as determined by previous titrations using tonsil and nasal polyp tissue) and left overnight ( $\geq 18$ hours) at $4^{\circ} \mathrm{C}$. Subsequently, the slides were brought to room temperature for $30 \mathrm{~min}$. The slides were washed with TBS and rabbit antimouse $2^{\mathrm{Y}}$ antibody at a dilution of 1:400 was applied for $30 \mathrm{~min}$. They were then washed with TBS before the application of a streptavadinbiotin biotin-peroxidase complex $(1: 1+75 \mu \mathrm{L}$ TBS) for $30 \mathrm{~min}$. The slides were then washed with TBS, and the chromagen diaminobenzidine (prepared in accordance with the manufacturer's instructions) was applied for $5 \mathrm{~min}$, before counterstaining with haematoxylin and placed under running tap water for $5 \mathrm{~min}$. The slides were then dehydrated, cleared and mounted.

The slides were analysed using the dotSlide virtual slide system (Olympus, Tokyo, Japan) and a blue 83-A Wratten filter $^{13}$ (Eastman Kodak, New York, USA). The filter was used to enhance the contrast for easier detection of stained cells of interest. Each layer of the bladder (mucosa, lamina propria, detrusor muscle and adventitia) was photographed five times, and the images were analysed using a cell counting programme routinely used to count the number of particles of interest. ${ }^{14}$ This programme, ImageJ, was used in conjunction with a macro that was developed by the group in order to identify positively stained MCs using an unbiased yet accurate method (figure 1).

The following guidelines were used to develop the automated cell counting method described above, in order to ensure unbiased, reproducible and reliable results:

- Only MCs that positively stained brown were counted.

- MCs that were on the edge of a photograph were only included if more than or equal to $50 \%$ was present in the image.

- If inadequate tissue for analysis was present, cross-pattern objective fields were selected at random.

Data were analysed using GraphPad Prism 6 (V.6.07; La Jolla, California, USA). Normality was tested by the Shapiro-Wilk method. Data were normally distributed; thus, statistical analysis was performed using multiple independent t-tests. In order to compare the density of each subtype of MC in the four layers of bladder wall that were analysed (adventitia, detrusor muscle, lamina propria and mucosa), individual paired t-tests comparing the average value of each of the layers against the average of a different layer, for each subtype, were conducted. Results were determined to be significant at 95\% CIs.

\section{RESULTS}

\section{Immunolocalisation of MCs within control and PBS/IC bladder} tissues

Immunostaining of tryptase containing MCs was moderate to intense, with staining exclusive to the cytoplasm and granules of MCs (figure 1). MCs that were actively degranulating during the time of tissue sampling have tryptase staining of granules beyond the outer membrane. AA1 positively identifies $\mathrm{MC}_{\mathrm{T}}$ and $\mathrm{MC}_{\mathrm{TC}}$ as these both contain tryptase, and CC1 positively identifies MC 


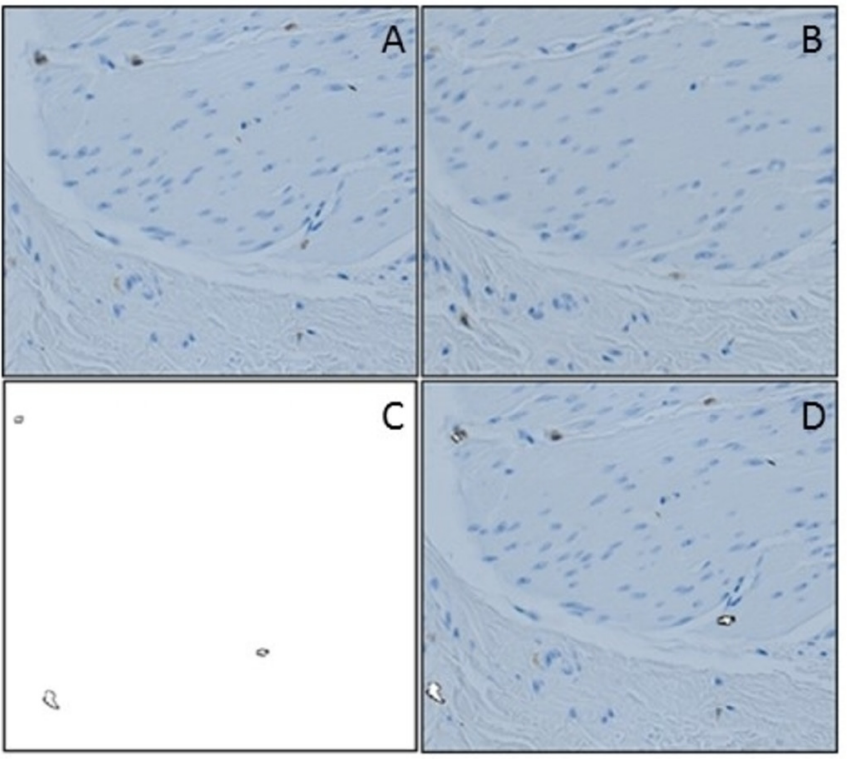

Figure 2 Schematic representation of CC1 positively stained mast cells among AA1 positively stained mast cells in PBS/IC bladder tissue. (A) AA1 positively stained mast cells in the detrusor layer of PBS/ IC bladder. (B) CC1 positively stained mast cells in the detrusor layer of PBS/IC bladder. (C) CC1 positively stained mast cells as detected by ImageJ processing of image (B). (D) Image A superimposed with image $C$ to show the distribution of CC1-positive mast cells as constituents of AA1-positive mast cells. PBS/IC, painful bladder syndrome/interstitial cystitis.

chymase, that is, $\mathrm{MC}_{\mathrm{TC}}$ only. Thus, some MCs will stain positively with both antibodies, and this is shown in figure 2 .

\section{Distribution of MC subtypes in control and PBS/IC bladder tissues}

AA1 and CC1 positive MCs show a similar pattern of distribution (figure 3 ).

\section{CC1-positive MCs}

In PBS/IC individuals, there was a statistically significant difference in the density of $\mathrm{MC}_{\mathrm{TC}}$ between the detrusor $(\mathrm{p}=0.01)$ and the lamina propria $(p=0.009)$ when compared with controls. $\mathrm{MC}$ proliferation was increased in PBS/IC tissue, with a difference in density related to location within the bladder wall (figure 3 ; average values \pm SEM).

\section{AA1-positive MCs}

Within the detrusor, the mean number of AA1-positive $\left(=\mathrm{MC}_{\mathrm{T}}+\right.$ $\mathrm{M}_{\text {СтС }}$ MC subtypes) MCs present in PBS/IC was 25.6 cells $/ 0.5 \mathrm{~mm}$ $\times 0.5 \mathrm{~mm}(\mathrm{n}=14)$ compared with only 2.7 cells $/ 0.5 \mathrm{~mm} \times 0.5 \mathrm{~mm}$ in the control group $(n=4)(p<0.001)$. In the lamina propria, the mean number of MCs found in PBS/IC was 21.5 cells $/ 0.5 \mathrm{~mm}$ $\times 0.5 \mathrm{~mm}(\mathrm{n}=14)$, compared with 1.7 cells $/ 0.5 \mathrm{~mm} \times 0.5 \mathrm{~mm}$ $(\mathrm{n}=4)$ in controls $(\mathrm{p}<0.001)$.

\section{Distribution of AA1-positive and CC1-positive MCs in PBS/IC and control bladder tissue ( \pm SEM bars)}

The average number of AA1 and CC1 positive MCs within the bladder wall of PBS/IC and control individuals is shown in figure 4. There is a similar pattern of distribution between the MC subtypes in PBS/IC tissue. Note the threefold increase in

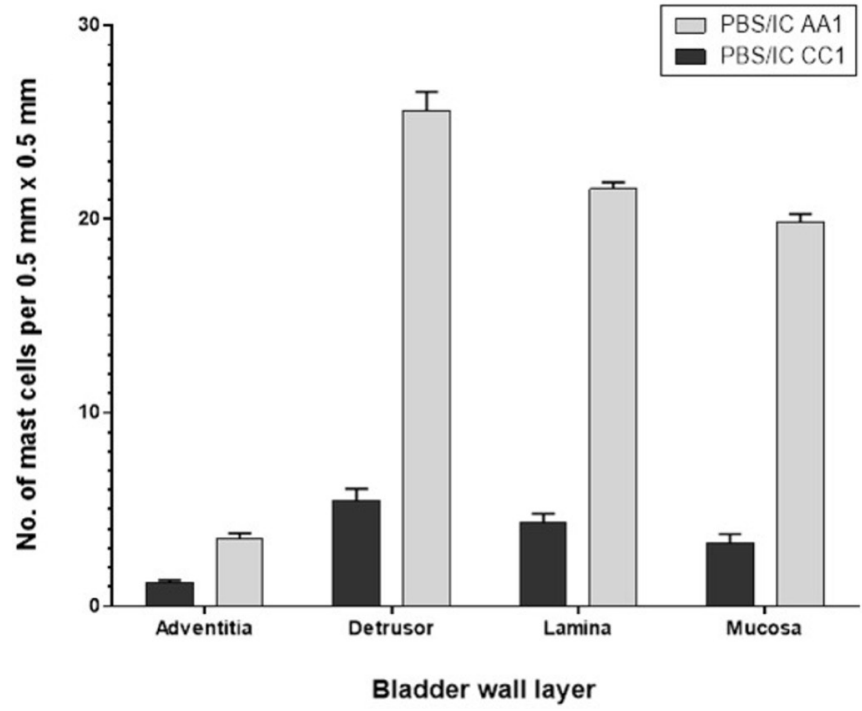

Figure 3 Distribution of AA1 positive and CC1 positive mast cells in PBS/IC ( \pm SEM bars). The average number of AA1 and CC1 staining mast cells within the bladder wall of PBS/IC individuals. As AA1 identifies all tryptase-containing mast cells (ie, both $\mathrm{MC}_{\mathrm{T}}$ and $\mathrm{MC}_{\mathrm{T}}$ ) and $\mathrm{CC} 1$ positive identifies $M C_{T C}$ only, the latter has been shown as a proportion of $A A 1$ identified mast cells. Note: there is a similar pattern of distribution between the mast cell subtypes in PBS/IC tissue. PBS/IC, painful bladder syndrome/interstitial cystitis.

the number of all MCs in the detrusor of subjects with PBS/IC, in comparison with controls. Supplementary information was provided in table 1 ; the mean numbers of $\mathrm{AA} 1\left(\mathrm{MC}_{\mathrm{TC}}\right.$ and $\left.\mathrm{MC}_{\mathrm{T}}\right)$ and $\mathrm{CC} 1\left(\mathrm{MC}_{\mathrm{TC}}\right)$ positive MCs were used to calculate the mean number of $\mathrm{MC}_{\mathrm{T}}$ cells in $\mathrm{PBS} / \mathrm{IC}$ bladder tissue.

\section{DISCUSSION}

MCs have long been considered as a key cell type in a range of inflammatory conditions and are currently thought to play a significant role in the development, persistence and pain associated with PBS/IC. ${ }^{911} 15$ Results from this study add further support to this hypothesis. Previous studies indicated that MC accumulation (mastocytosis) was only present in a subset of patients. ${ }^{15-17}$ In this study, mastocytosis was present in all individuals with $\mathrm{PBS} / \mathrm{IC}$. However, it must be noted that the samples were taken from a group of PBS/IC patients with more severe and advanced disease.

In the present study, we observed differences in the distribution and density of MCs within the bladder wall between normal and PBS/IC bladders, with the difference in MC density within the detrusor being statistically significant $(\mathrm{p}<0.001)$. This is in addition to the differences in MC distribution seen in the layers of PBS/IC bladder. Generally, both MC subtypes are located close to blood vessels within the lamina and close to nerve fibres within the detrusor. This difference in the location of MC accumulations supports the suggested pathology of PBS/IC: that MC recruitment and degranulation is triggered by inflammatory factors. ${ }^{10}$ The statistically significant increase in $\mathrm{MC}$ density within the detrusor of PBS/IC may be attributed to the increase in inflammatory cytokines such as SCF. Activated MCs also release various factors such as SP. Thus, the detrusor muscle mastocytosis seen in this study can be explained by a self-perpetuating cycle of $\mathrm{MC}$ activation and recruitment.

The results also indicate that although there is no significant difference in the density of MC subtypes in controls (figure 4), 


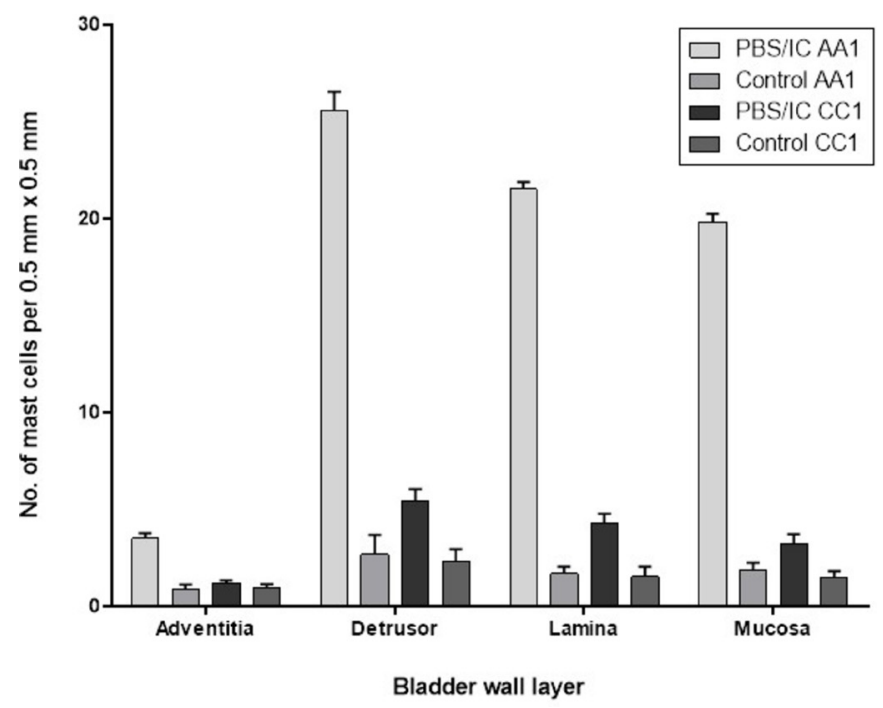

Figure 4 Distribution of AA1-positive and CC1-positive mast cells in PBS/IC and control bladder tissue ( \pm SEM bars). The average number of AA1 and CC1 staining mast cells within the bladder wall of PBS/IC and control individuals. Note the threefold increase in the number of AA1-positive mast cells in the detrusor muscle layer of subjects with $\mathrm{PBS} / \mathrm{IC}$, in comparison with controls. PBS/IC, painful bladder syndrome/ interstitial cystitis.

the increased density of both subtypes within the detrusor supports the currently accepted pathogenesis of PBS/IC as discussed above. This may explain why the majority of current treatment options have a success rate of $61 \%{ }^{18}$ or less; these therapies are failing to target the stimulation of nerve fibres, which occurs when urinary components leak through a damaged GAG layer.

The significant increase of the $\mathrm{MC}_{\mathrm{TC}}$ subtype in the detrusor $(p=0.01)$ and lamina $(p=0.009)$ of individuals with PBS/ IC is an interesting finding, as chymase has a greater destructive potential than tryptase and may explain the variability in severity of symptoms between PBS/IC sufferers. The number of $\mathrm{MC}_{\mathrm{TC}}$ within the adventitia is almost identical between normal and PBS/IC bladder. This suggests that $\mathrm{MC}_{\mathrm{TC}}$ is a constitutive component of the bladder and that the larger influx of $\mathrm{MC}_{\mathrm{T}}$ is the more important event in PBS/IC (figure 3). MC subtypes have different roles within the body; $\mathrm{MC}_{\mathrm{T}}$ are associated with the immune system and are found predominantly at mucosal surfaces, and $\mathrm{MC}_{\mathrm{TC}}$ are non-immune system associated and are found predominantly in connective tissue.

As $\mathrm{MC}_{\mathrm{TC}}$ are increased in fibrotic disease, it could be suggested that the statistically significant influx of $\mathrm{MC}_{\mathrm{TC}}$ in the lamina propria and detrusor of PBS/IC bladder may be linked to a higher risk of fibrosis. Clinically, this would be reflected

\begin{tabular}{|c|c|c|c|}
\hline Bladder wall layer & $\mathrm{AA} 1\left(\mathrm{MC}_{\mathrm{TC}}\right.$ and $\left.\mathrm{MC}_{\mathrm{T}}\right)$ & $\mathrm{CC} 1\left(\mathrm{MC}_{\mathrm{TC}}\right)$ & $\mathrm{AA1}-\mathrm{CC} 1\left(\mathrm{MC}_{\mathrm{T}}\right)$ \\
\hline Adventitia & 3.529 & 1.217 & 2.312 \\
\hline Detrusor & 25.57 & 5.467 & 20.10 \\
\hline Lamina & 21.54 & 4.317 & 17.22 \\
\hline Mucosa & 19.84 & 3.267 & 16.57 \\
\hline
\end{tabular}

The mean number of AA1-positive ( $\mathrm{MC}_{\mathrm{TC}}$ and $\mathrm{MC}_{\mathrm{T}}$ ) and $\mathrm{CC} 1$-positive $\left(\mathrm{MC}_{\mathrm{TC}}\right.$ ) mast cells within the layers of the bladder wall of individuals with $\mathrm{PBS} / \mathrm{IC}$. These figures have been used to calculate the number of $M C_{T}$ in $P B S / I C$.

$\mathrm{PBS} / \mathrm{IC}$, painful bladder syndrome/interstitial cystitis.

\section{Take home messages}

- The increase in mast cells is of key importance, and the difference between subtypes may explain the variability seen in interstitial cystitis symptomology and reflect disease severity.

- These findings could potentially be used to develop more comprehensive diagnostic criteria and novel tailored therapeutic options for interstitial cystitis patients.

as a low bladder capacity due to thickening and stiffening of the bladder wall. To confirm this, further histological staining of bladder wall tissue samples would be required. In addition, on unblinding to the patient data and diagnostic notes, a link between a substantial presence of $\mathrm{MC}_{\mathrm{TC}}$ in the mucosa and the presence of ulcers was observed. This novel finding may have a significant clinical ramifications; that is, it would allow the identification of patients who suffer from the less common Hunner's ulcer subtype of PBS/IC. ${ }^{12}$

The main limitation of the study is that the number of patients in both test groups was small. Only full-thickness bladder sections were selected, and this type of tissue sample is less likely to be taken during cystoscopy. Although the paraffin-embedded tissue block may have been classed as full-thickness in the tissue bank database, once the tissue had undergone processing, paraffinisation, storage and preparation for this study, shrinkage and tissue loss occurred, thus resulting in incomplete representation of the bladder wall. Consequently, a number of patients were excluded from the study.

In conclusion, PBS/IC is an under-researched disorder that remains poorly understood, diagnosed and managed. The study highlights the role of MCs in the diagnosis of PBS/IC. A diagnosis of PBS/IC is based on the patient's symptoms and urological assessment, including cystoscopy and exclusion of other common urinary diseases such as bacterial cystitis, urethritis and neoplasia. ${ }^{19}$

Despite this, only the European Society for Study of Interstitial Cystitis (ESSIC) criteria incorporate the presence of detrusor mastocytosis or inflammatory infiltrates for PBS/IC diagnosis. This study and other similar work suggest that the MC count could be incorporated into official diagnostic criteria. Although not all patients exhibit mastocytosis, inclusion of a minimum MC count would aid the identification of individuals who are otherwise misdiagnosed. MC subtyping might also help distinguish between cases of PBS/IC of differing severity. Following further confirmation, these findings could be used to develop muchneeded comprehensive diagnostic criteria for the successful identification of PBS/IC sufferers, and for the development of novel tailored therapeutic options including strategies to inhibit MC degranulation, that would benefit patient management.

Handling editor Cheok Soon Lee.

Contributors STM: made a substantial contribution to the design, organisation and conduct of the study (including data acquisition). BRB: made a substantial contribution to the conception and critiquing the output for important intellectual content. DV, MF, VF, AJC and AFW: made substantial contribution to data analysis, manuscript preparation revision and critiquing the output for important intellectual content. BAL: made a substantial contribution to the conception, design, organisation and conduct of the study, including manuscript preparation and revision.

Funding The authors have not declared a specific grant for this research from any funding agency in the public, commercial or not-for-profit sectors.

Competing interests None declared. 
Patient consent Not required.

Provenance and peer review Not commissioned; externally peer reviewed.

(C) Article author(s) (or their employer(s) unless otherwise stated in the text of the article) 2018. All rights reserved. No commercial use is permitted unless otherwise expressly granted.

\section{REFERENCES}

1 Bassi PF, Costantini E, Foley S, et al. Glycosaminoglycan Therapy for Bladder Diseases: Emerging New Treatments. European Urology Supplements 2011;10:451-9.

2 Metcalfe DD. Mast cells and mastocytosis. Blood 2008;112:946-56.

3 Sant GR, Kempuraj D, Marchand JE, et al. The mast cell in interstitial cystitis: role in pathophysiology and pathogenesis. Urology 2007;69(4 Suppl):S34-S40.

4 Parsons CL. The role of a leaky epithelium and potassium in the generation of bladde symptoms in interstitial cystitis/overactive bladder, urethral syndrome, prostatitis and gynaecological chronic pelvic pain. BJU Int 2011;107:370-5.

5 Bjorling DE, Jerde TJ, Zine MJ, et al. Mast cells mediate the severity of experimental cystitis in mice. J Urol 1999:162:231-6.

6 Bouchelouche K, Alvarez S, Horn T, et al. Human detrusor smooth muscle cells release interleukin-6, interleukin-8, and RANTES in response to proinflammatory cytokines interleukin-1 beta and tumor necrosis factor-alpha. Urology 2006;67:214-9.

7 Christmas TJ, Rode J, Chapple CR, et al. Nerve fibre proliferation in interstitial cystitis. Virchows Arch A Pathol Anat Histopathol 1990;416:447-51.

8 Pang $X$, Marchand J, Sant GR, et al. Increased number of substance $P$ positive nerve fibres in interstitial cystitis. Br J Urol 1995;75:744-50.
9 McCary C, Tancowny BP, Catalli A, et al. Substance P downregulates expression of the high affinity IgE receptor (FcepsilonRI) by human mast cells. J Neuroimmunol 2010;220(1-2):17-24.

10 Theoharides TC, Alysandratos KD, Angelidou A, et al. Mast cells and inflammation Biochimica et Biophysica Acta (BBA) - Molecular Basis of Disease. 2012;1822:21-33.

11 Peeker R, Enerbäck L, Fall $M$, et al. Recruitment, distribution and phenotypes of mast cells in interstitial cystitis. J Urol 2000;163:1009-15.

12 Yamada T, Murayama T, Mita H, et al. Subtypes of bladder mast cells in interstitial cystitis. Int J Urol 2000;7:292-7.

13 Møller M, Glistrup OV, Olsen W. Contrast enhancement of the brownish horseradish peroxidase-activated 3,3'-diaminobenzidine tetrahydrochloride reaction product in black and white photomicrography by the use of interference filters. $J$ Histochem Cytochem 1984;32:37-42.

14 Yeo JC, Wall AA, Stow JL, et al. High-throughput quantification of early stages of phagocytosis. Biotechniques 2013;55:115-24.

15 Theoharides TC, Kempuraj D, Sant GR. Mast cell involvement in interstitial cystitis: a review of human and experimental evidence. Urology 2001:57:47-55.

16 Roth TM. Interstitial cystitis in a woman with systemic mastocytosis. Int Urogynecol J Pelvic Floor Dysfunct 2007:18:963-5.

17 Rössberger J, Fall M, Gustafsson CK, et al. Does mast cell density predict the outcome after transurethral resection of Hunner's lesions in patients with type $3 \mathrm{C}$ bladder pain syndrome/interstitial cystitis? Scand J Urol Nephrol 2010;44:433-7.

18 Stav K, Beberashvili I, Lindner A, et al. Predictors of response to intravesical dimethylsulfoxide cocktail in patients with interstitial cystitis. Urology 2012;80:61-5.

19 Nigro DA, Wein AJ, Foy M, et al. Associations among cystoscopic and urodynamic findings for women enrolled in the Interstitial Cystitis Data Base (ICDB) Study. Urology 1997;49:86-92. 